\title{
The spatial representation of chemical structures in the antennal lobe of honeybees: steps towards the olfactory code
}

\author{
Silke Sachse, Angelika Rappert and C. Giovanni Galizia \\ Institut für Neurobiologie, Fachbereich Biologie, Freie Universität Berlin, D-14195 Berlin, Germany \\ Keywords: Apis mellifera, hydrocarbons, molecular receptive range, olfactory glomeruli, optical recording
}

\begin{abstract}
Odours are represented by specific ensembles of activated glomeruli in a combinatorial manner within the olfactory bulb of vertebrates or the antennal lobe $(\mathrm{AL})$ of insects. Here, we optically measured glomerular calcium activities in vivo in the honeybee Apis mellifera during olfactory stimulation with 36 pure chemicals differing systematically in carbon chain length $(C-5-10)$ and functional group (aldehyde, ketone, alcohol, carboxylic acid and alkane). We show their glomerular representations in 38 morphologically identified glomeruli out of the honeybee's 160 . We measured the molecular receptive range of identified glomeruli averaging up to 21 individuals. Of the 38 glomeruli measured, 23 show maximal activity in a specific range of chain length. Glomeruli preferentially responding to a functional group are also always broadly tuned to particular chain lengths. Furthermore, glomeruli with similar response spectra are often direct neighbours. The results allow conclusions about the interactions between olfactory receptors and odour molecules, and about the AL network.
\end{abstract}

\section{Introduction}

For the honeybee Apis mellifera the olfactory system is essential in recognizing, classifying and learning a vast number of odours (Frisch, 1967). Many of these odours are composed of hydrocarbons, e.g. alcohols and aldehydes (Dobson, 1994).

In the honeybee 60000 olfactory receptor neurons (ORNs) (Esslen $\&$ Kaissling, 1976) innervate the first olfactory neuropil, the antennal lobe (AL), through four tracts (T1-T4) (Suzuki, 1975). The AL is the structural and functional analogue of the olfactory bulb of vertebrates (Boeckh et al., 1990). Both have a glomerular structure and process incoming signals from broadly tuned ORNs (Boeckh et al., 1990; Shepherd, 1991). The single AL consists of 156 glomeruli arranged in a single layer around the AL. Almost all the synapses between ORNs, 4000 local interneurons (LINs) and 800 outgoing projection neurons (PNs) are located within the glomeruli (Gascuel \& Masson, 1991). Glomeruli have characteristic shapes and orientations which make them identifiable using a three-dimensional atlas of the honeybee AL (Flanagan \& Mercer, 1989; Galizia et al., 1999a). Each glomerulus is given a name consisting of the tract of the antennal nerve from which it receives input and a number, e.g. T1-46 or T3-46.

It is possible to optically record odour-evoked activities in the olfactory bulb (Cinelli et al., 1995; Friedrich \& Korsching, 1997, 1998) or in the AL (Joerges et al., 1997; Galizia et al., 1997, 1998). Odours lead to a specific spatio-temporal pattern of activated glomeruli (Joerges et al., 1997; Friedrich \& Korsching, 1997) which is bilaterally symmetrical (Galizia et al., 1998) and species specific (Johnson et al., 1998; Galizia et al., 1999b). Therefore, each glomerulus has a particular olfactory receptor range.

Correspondence: S. Sachse, as above.

E-mail: silsis@zedat.fu-berlin.de

Received 4 May 1999, revised 8 July 1999, accepted 16 July 1999
The extraordinary discriminative capacity of bees, as shown in behavioural experiments (Vareschi, 1971), suggests a distinct representation in the $\mathrm{AL}$, even for closely related chemicals. Results from single-cell measurements of mitral cells in the olfactory bulb of vertebrates show that aldehydes of similar chain length are represented in neighbouring areas (Mori et al., 1992), and that similarities in stereochemical structures of the odorants are strongly correlated to the tuning specificity of mitral cells (Imamura et al., 1992). Using radioactively labelled 2-deoxyglucose in rats, Johnson et al. (1998) have shown that functional groups of the odorants activate specific areas in the bulb. This would suggest that single glomeruli represent particular chemical or structural properties or features, in a way similar to what is often assumed about olfactory receptor proteins.

We measured the responses of 38 identified glomeruli to a range of hydrocarbons differing systematically in carbon chain lengths and functional groups. The results show that the glomerular receptive range mainly depends on carbon chain length. We found no glomeruli which preferentially respond to functional groups independently of chain length. The position of functional groups of alcohols influences the chain length coding. Parts of this work have been published in abstract form (Sachse et al., 1998).

\section{Materials and methods}

\section{Preparation and staining process}

Adult worker honeybees were caught from four different hives, which were kept in a flight chamber at a constant night/day rhythm. After cooling, the bee was fixed in a Plexiglas chamber using dental wax. The antennae were restrained from moving with two silicone components (Kwik-Sil ${ }^{\mathrm{TM}}$, WPI) at their scapus. A window was cut into the cuticle of the head. Glands and tracheae were removed; the 
oesophagus was cut. The brain was then washed in Ringer solution (in $\mathrm{mM}: \mathrm{NaCl}, 130 ; \mathrm{KCl}, 6 ; \mathrm{MgCl}_{2}, 4 ; \mathrm{CaCl}_{2}, 5$; sucrose, 160 ; glucose, 25; HEPES, 10, pH 6.7, $500 \mathrm{mOsmol}$ ) in order to remove proteases and other enzymes that may have been released by glands or by the cut oesophagus. Fifty microlitres of dye, Calcium Green 1or 2-AM (Molecular Probes, Eugene, USA) was first dissolved in $50 \mu \mathrm{L}$ Pluronic in dimethylsuphoxide (DMSO) and then diluted in $950 \mu \mathrm{L}$ Ringer solution. The brain was exposed and stained using a bath application. After $1 \mathrm{~h}$ of staining the brain was washed with Ringer solution in order to remove excess dye and the abdomen was cut.

\section{Optical imaging and stimulus delivery}

In vivo calcium recordings have been performed as described elsewhere (Galizia et al., 1998). All measurements were performed between January and March 1998. The stained bees were placed under an epifluorescent microscope $(20 \times$, NA 0.6 air obj.) with constant Ringer perfusion $(1 \mathrm{~mL} / \mathrm{min})$ at room temperature $\left(22^{\circ} \mathrm{C}\right)$. For each odour, $4 \mu \mathrm{L}$ of the pure substance were placed on a filter paper $\left(1 \mathrm{~cm}^{2}\right)$ in a $1-\mathrm{mL}$ plastic syringe. The stimuli were delivered by manually puffing $0.8 \mathrm{~mL}$ of odour-laden air (duration, $2 \mathrm{~s}$ ) into a constant air stream. The control stimulus was a syringe plus filter paper without odour. The odour concentrations used do not saturate the calcium signal, and are routinely used in behavioural assays in our laboratory. Different concentration levels (see, e.g. Fig. 4 below) were produced by a dilution of the odour in $n$-hexane. All odour syringes were emptied and kept in a clean air stream over night, and loaded with new filter paper and odour the next day. The odours were stored in flasks at $5{ }^{\circ} \mathrm{C}$. The chemical purities were checked with a GC-analysis after the whole experiment, and found to be $>99 \%$. All odours were from Sigma-Aldrich, Deisenhofen, Germany. Images were taken at a rate of $\sim 2$ frames/s, with $240 \mathrm{~ms}$ exposure time per image and measured with a 12-bit CCD camera (Photometrics CH250A, Tucson, AZ, USA). The interstimulus interval was $45 \mathrm{~s}$. Between 40 and 80 measurements were performed in each bee. We measured the response patterns in a total of 24 bees (each bee was tested with a subset of odours).

\section{Mapping of glomeruli}

The glomerular structure is not visible during calcium imaging. Therefore, after the calcium measurements the neurolemma was digested with protease (Protease Type XIV, Sigma) for $5 \mathrm{~min}$, and then the AL was stained with the membrane-soluble dye RH795 (Molecular Probes). After a period of $\sim 30-45 \mathrm{~min}$ the glomerular structure was visible in epifluorescent light and imaged at different focus levels (Fig. 1A, interplane distance $10 \mu \mathrm{m}$ ). The images were sharpened with Photoshop (Adobe) in order to reconstruct the glomerular borderlines for each measured animal. The glomeruli were identified with a computer atlas of the AL (Galizia et al., 1999a) by comparing their morphology and position with those of other glomeruli (Fig. 1B). In this preparation, the frontal view of the $\mathrm{AL}$ was visible. Most glomeruli in this region receive input by the $\mathrm{T} 1$ tract from the antennal nerve. In every preparation we could identify between 23 and 36 glomeruli. Each reconstructed glomerulus was eroded by 1 pixel (i.e. its area reduced) and projected onto the activity patterns. Excitation of single glomeruli in response to each odour stimulus was calculated by averaging the response of all pixels belonging to the glomerulus.

\section{Response quantification and statistical analysis}

Relative fluorescent changes $(\Delta F / F)$ were calculated from the raw data as in Galizia et al. (1998). Therefore, the time courses (blue line in Fig. 1C) were corrected for bleaching using a linear regression computed through the mean values of the intervals defined by frames 4-9 and 20-25 (blue dashed line and white dotted lines in Fig. 1C), and by subtracting the air control (white line in Fig. 1C). The integral over the time interval defined by frames 10-19 (Fig. 1D) was taken as the glomerular activation $\left(R_{\mathrm{int}}\right)$, thus disregarding any dynamical aspects of the responses. We took the median response of up to three measurements as that animal's response to that stimulus. Examples for signals from identified glomeruli to different odour stimulations are shown in Fig. 1E-G. For the calculation of the glomerular olfactory code (Fig. 2), we set the strongest glomerular response $\left[R_{\text {int }}(\max )\right]$ of each animal at $100 \%$ and scaled the other responses accordingly. We categorized these normalized responses into five equal bins: noise to $20 \% ; 20-40 \% ; 40-60 \% ; 60-80 \% ; 80-100 \%$. Noise was estimated as the average standard deviation of the response in the interval covering frames 4-9 (i.e. before stimulus). This procedure yields the relative response pattern to an odour, disregarding the absolute response magnitude. Variability between individuals was estimated by calculating the standard deviation (SD) of the normalized data; this statistic is termed 'variability range' in the text.

For the comparison of absolute glomerular responses to different carbon chain lengths (e.g. Fig. 5A) and functional groups (e.g. Fig. $6 \mathrm{~A}$ ) we set the response of glomerulus T1-28 to 1-hexanol to $100 \%$ in each measured individual and scaled the other responses accordingly. This procedure allows the comparison of absolute responses between individuals.

For the calculation of the cumulative AL activity (Fig. 3A) only glomeruli with big sampling sizes were included. These were for T1: $17,18,23,24,25,28,29,30,33,35,36,37,38,39,42,43,47,48,49$, 52, 60 and 62; for T3: 31 and 45 (24 glomeruli). The values correspond to those given in Fig. 2, multiplied by the corresponding $R_{\text {int }}(\max )$. The sum over these glomeruli gives the cumulative $\mathrm{AL}$ activity.

The vapour pressures of the different odours tested in this study were estimated from their boiling points using the Hass and Newton equation (Hass \& Newton, 1975). Using the linear relation between the amount of odorant loaded and odorant released from filter paper (Kaissling, 1995), we calculated an approximation of the effective vapour pressure for different odour concentration levels.

Statistical analysis was performed using JMP 3.2.1, Statistical Discovery Software (SAS; Cary, NC, USA) based on the normalized responses. We excluded all odours with a maximum absolute response below $R_{\text {int }}=2.5$ (Fig. 2), i.e. decan, undecan, dodecan, tridecan and all carboxylic acids.

Each odour representation can be regarded as a vector in a multidimensional space, where each glomerulus represents one dimension. Because it is not possible to visualize points in an 18dimensional space, we used a principal component analysis (PCA). This technique calculates a new cartesian coordinate system based on the eigenvectors of the correlation matrix in which the first axis (i.e. the first principal component) explains most of the variance observed, the second most of the remaining variance, etc. It therefore allows the visualization of the relative positions of the points observed in a reference system with reduced dimensionality. We included a varimax rotation of the first three principal components in order to make the factors more interpretable. The varimax method tries to make elements of this matrix go towards 1 or 0 to show the clustering of variables. In order to minimize missing data in the matrix of the PCA (e.g. Figs 5 and 6 below), glomeruli were only included if they had $<20$ missing entries for all odours. This left the following 18 glomeruli: for T1: 17, 23, 24, 25, 28, 29, 33, 35, 36, 37, 38, 42, 43, 47, 48, 49 and 60; for T3: 45 . Missing cells (53 of 4716 entries $=1.1 \%$ ) 

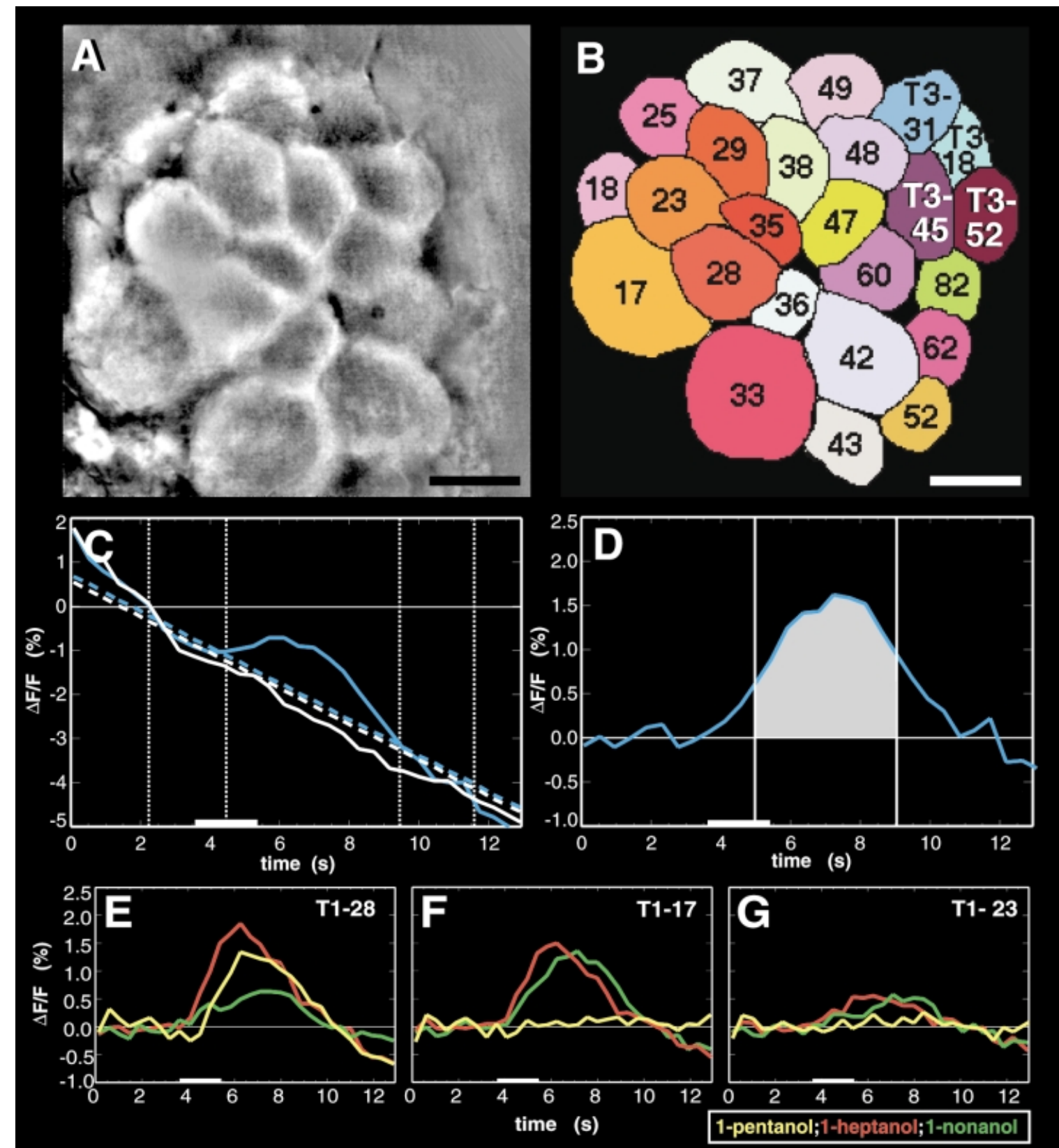

FIG. 1. Glomerular identification, data evaluation and time courses in calcium-imaging studies of the honeybee AL. (A) View of the AL after staining with RH795. The boundaries of the glomeruli are visible. Images like this from several focal levels can be combined to reconstruct the glomerular arrangement. Scale bar, $50 \mu \mathrm{m}$ (40 $\times$ objective). (B) Reconstruction of the glomerular map for the AL shown in A. The single glomeruli have been identified with the aid of the digital AL atlas. Each glomerulus is shown with a unique, arbitrary colour. All glomeruli without tract labelling are innervated by T1. Scale bar, $50 \mu \mathrm{m}$. (C) Time courses of 1 pixel of a glomerulus during air (white line) and odour application (blue line). Because the amount of bleaching is not equal in all trials, the raw data were corrected using a linear regression (white and blue dashed lines) computed through the mean values of the time intervals corresponding to frames 4-9 and frames 20-25 (marked by the dotted lines) and then subtracting the air control. (D) Single time course of the same pixel as shown in C after linear correction and air subtraction (blue line). We calculated the integrated response $R_{\text {int }}$ between frames 10 and 19 inclusive (labelled area). (E-G) Time traces of the responses of three identified glomeruli to three primary alcohols (1-pentanol, 1-heptanol, 1-nonanol) plotted in $\Delta F / F$ over time. The white bar marks the stimulus. Note the different response characteristics. Glomerulus T1-28 reveals a strong response to 1-pentanol, intermediate response to 1-heptanol and only a weak response to 1-nonanol. The responses of glomerulus T1-17 show the opposite behaviour. Glomerulus T1-23 reveals only low activation.

were replaced with the within-group mean. The PCA of alcohol responses was performed on all measurements of single individuals. For the PCA of functional groups only the averaged species responses were used. The correlation matrix (Table 1) included the same glomeruli as the calculation of the cumulative AL activity. For the comparison between odours with identical functional groups but with neighbouring carbon chain lengths we averaged the correlations of the primary alcohols, aldehydes and secondary ketones.

\section{Results}

\section{The glomerular response pattern for hydrocarbons}

We measured the molecular receptive range of 38 identified glomeruli using calcium imaging of the honeybee antennal lobe. We screened 36 hydrocarbons as olfactory stimuli that differed systematically in carbon chain lengths and functional groups. Circle sizes in Fig. 2 represent the responses to odours averaged over all 


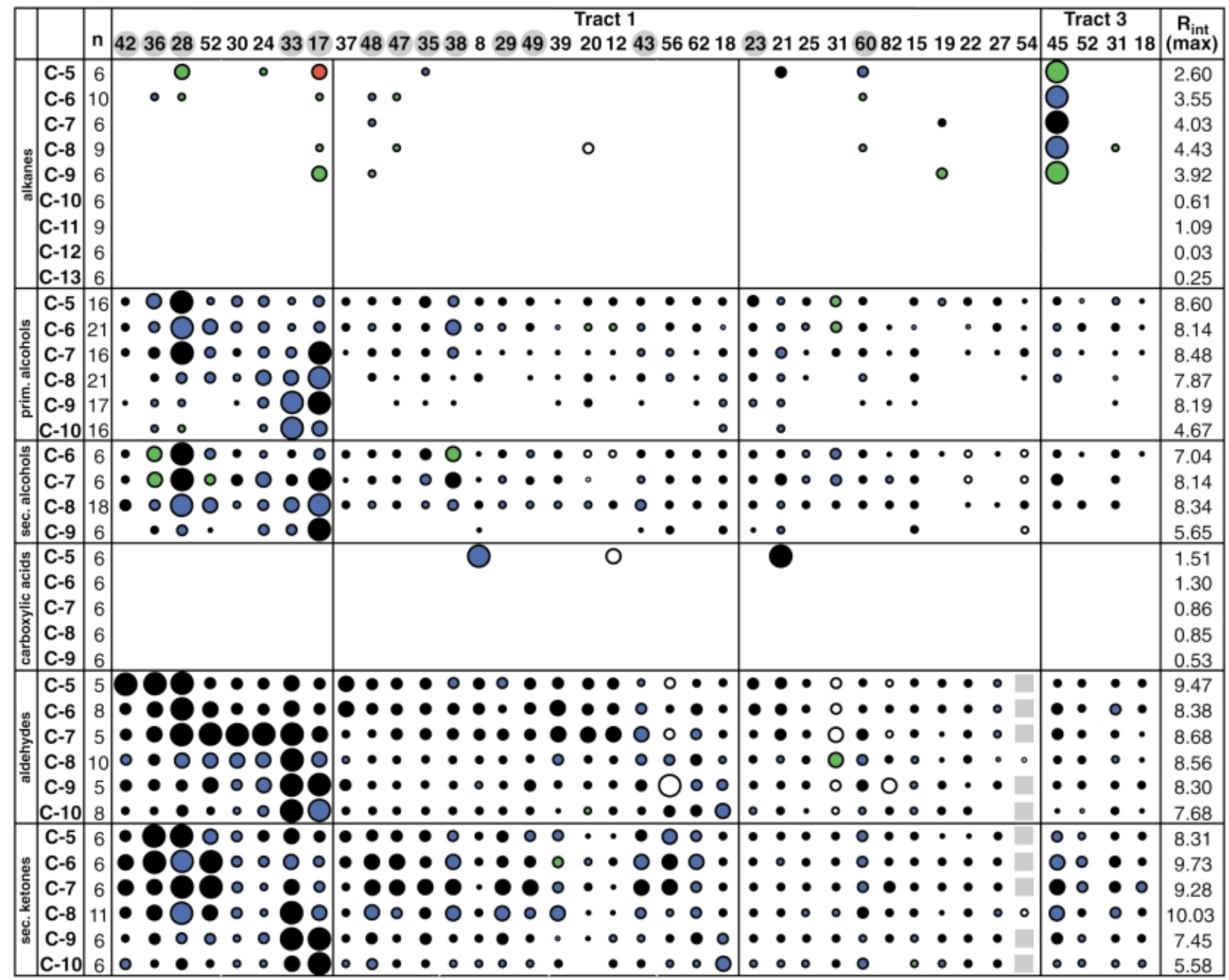

response (circle size):

O $80-100 \%$; O $60-80 \%$; 0 40-60\%; ० 20-40\%; •noise -20\%; <noise; no measurement

variability range (color, white circle: $n=1$ ):

s.d. $<15 \%$; s.d. $<25 \%$; s.d. $<35 \%$; s.d. $<45 \%$

FIG. 2. The olfactory code of honeybee glomeruli. Each row represents one odour, each column an identified glomerulus. The odorants are arranged according to their chemical structures and with increasing carbon chain lengths. The glomeruli are sorted in three groups for T1 and one group for T3. The first group of T1 glomeruli show strong, the second intermediate and the third no response properties influenced by chain length. For T3, only glomerulus 45 reveals strong chain length dependence. The glomerular response intensity is coded by circle size in five bins. Empty entries indicate responses below noise limits. Missing measurements are marked with a grey box. The circle's colour filling indicates the variability range between individuals as the standard deviation (s.d.). White fillings show measurements of only one animal. The number of measurements (n) applies to the glomeruli marked with the grey circles in the title line. The other glomeruli could only be recognized in a subset of animals. The right column $\left[R_{\text {int }}(\max )\right]$ gives the maximum response to that odour, averaged over all individuals. The activity patterns are specific and nearly constant between individuals for each odour.

measured animals. The colours of the entries represent their consistency between individuals. Glomeruli with dominant responses to specific odours reveal a strong consistency; $71 \%$ of the 47 entries with the strongest response have a variability range (SD/s.d.) lower than $15 \%, 24 \%$ have a variability range between 15 and $25 \%$, and $4 \%$ have a variability range between 25 and $35 \%$. Only one entry has a variability range higher than $35 \%$.

Figure 2 displays the glomerular activity patterns for each odour. The maximum response $\left[R_{\mathrm{int}}(\max )\right]$ and the number of activated glomeruli are dependent on the odour. Consequently, the cumulative AL activity (see Materials and methods) is odour specific. For example, heptanal, octanal, 2-hexanone and 2-heptanone elicit strong glomerular responses, while alkanes of 10 carbon atoms or more and carboxylic acids of six atoms or more cause activities only within noise limits. Figure 3A depicts the relationship between chemical structure and cumulative AL activity. A decrease in the cumulative AL activity is apparent with the following odour succession: ketones, aldehydes, secondary and primary alcohols, alkanes and carboxylic acids. Activity generally decreases with increasing chain length within a chemical family (Fig. 3A). The vapour pressure reflects the number of molecules per stimulus, and also decreases with increasing chain length (Fig. 3B). Therefore, the decrease in activity of the 38 glomeruli measured could be due to the reduced vapour pressure. Indeed, for all odours (apart from alkanes and carboxylic acids) the correlation between vapour pressure and activity is very pronounced (Fig. 3C).

In addition to the stereochemistry, the electronegativity of the odour molecule might be an important factor in odour-receptor 

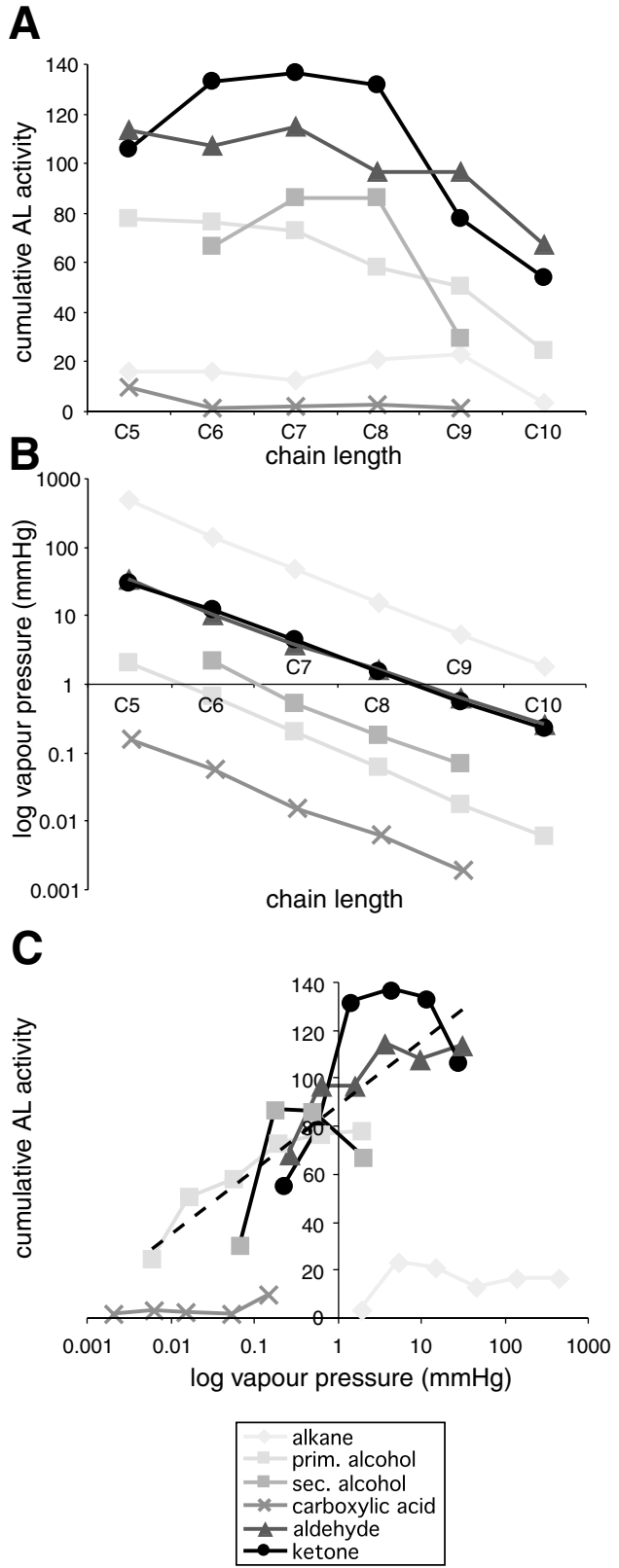

FIG. 3. Cumulative glomerular activity and vapour pressure of hydrocarbons for different carbon chain lengths. (A) Plot of cumulative AL activity against carbon chain length for the five chemical classes investigated. Cumulative glomerular responses decrease from the ketones to the carboxylic acids. (B) Plot of logarithm vapour pressure against carbon chain length for the five chemical classes investigated. The vapour pressure is inversely proportional to carbon chain length. Alkanes show the strongest, carboxylic acids the lowest volatility. Aldehydes and secondary ketones are not distinguishable in relation to the vapour pressure. (C) Plot of cumulative AL activity against logarithm vapour pressure for the different hydrocarbons investigated. Both variables show a nearly linear relationship, with the exception of responses to alkanes and carboxylic acids. The regression line (dashed line) is based on the data of the alcohols, aldehydes and ketones. Note that alkanes elicit a very weak glomerular activation but have the strongest vapour pressures.

interaction and thus in glomerular activation. The inductive substitute constant $\sigma^{*}$ can be used as a measure for the electronegativity of a functional group; $\sigma^{*}$ decreases with the following succession: aldehydes (2.04), ketones (1.65), carboxylic acids (0.95), primary and secondary alcohols ( 0.56 and 0.46 , respectively) and alkanes
(0.0) (Hauptmann, 1987). Indeed, alkanes have no electronegativity and elicit very low responses in the 38 glomeruli measured. However, stimulus concentration is a better predictor for the cumulative activities of the other chemical classes than electronegativity, and the response to alkanes in T3-45 (Fig. 2) is certainly not a function of electronegativity. This contrasts with vertebrates, where it has been shown that the sensitivity of ORNs increases for functional groups with higher electronegativity (Sato et al., 1994).

\section{Specificity of activity patterns}

Decreasing vapour pressure leads to a weak overall response, but does not interfere with the spatial pattern of relative activity. Measurements of glomerular activity at three different concentrations show consistent glomerular activation (Fig. 4A). The cross-wires mark the glomerulus T1-28 with the strongest response to 1-pentanol (compare with Fig. 2). This glomerulus is the strongest for all concentrations of pentanol tested. Glomeruli with intermediate activity show reduced responses or disappear at lower concentrations. The activity patterns elicited by 1-heptanol and 1-nonanol reveal similar properties. Because in non-saturated ranges the release of odorant from a filter paper is proportional to the amount of odorant loaded (Kaissling, 1995), we were able to estimate the relative quantity of odour molecules in different stimuli from their vapour pressure. A concentration of $1 \%$ for 1-pentanol corresponds to concentrations of $10 \%$ for 1-heptanol and $100 \%$ for 1-nonanol, respectively. The responses to these concentrations are marked with coloured borders in Fig. 4A. Comparing the responses of the two identified glomeruli T1-28 and T1-17 to identical odour concentrations (symbols in Fig. 4B), it is apparent that the glomerular receptive range is concentration independent over three orders of magnitude, and corresponds to the averaged response profiles (Fig. 5A).

\section{Carbon chain length influences the response to hydrocarbons}

The response of four identified glomeruli during stimulation with primary alcohols that differ in their carbon chain length is shown in Fig. 5A. Maximum glomerular responses are restricted to a specific chain length range. Glomerulus T1-28 is strongly activated by short carbon chains. Glomeruli T1-17 and T1-52 reveal maximal activity when stimulated with an intermediate chain, and an increase in activity for longer hydrocarbons is apparent for glomerulus T1-33 (compare with time courses for T1-17 and T1-28 in Fig. 1E and F). Consideration of all chemical classes tested shows that 23 of the 38 glomeruli measured reveal maximal activity in a specific range of chain length, irrespective of the functional group of the chemical used as stimulus (Fig. 2). Most of these responses have their maximum range at an intermediate chain length (10 glomeruli: T1-20, 24, 29, 30, 39, 43, 49, 52, 62 and T3-45); the others are either maximally stimulated by short chain length (seven glomeruli: T1-28, 35, 36, 38, 42, 47 and 48) or at longer carbon chains (three glomeruli: T1-17, 18 and 33). While the optimal chain length is not necessarily equal for different functional groups, it never differs by more than three carbon atoms. For example, the response maximum of glomerulus T1-17 to different functional groups varies between C-7 and C-10 (Fig. 2). This indicates coding differences between the chemical classes (see below).

A PCA (for details see Materials and methods) of the multidimensional space spanning the single animal responses to primary alcohols reveals that most of the variance $(69.8 \%)$ can be represented in a three-dimensional space (Fig. 5B). For clarity these three dimensions are shown in two-dimensional projections. The eigenvectors are a measure for the contribution of each factor (here of each glomerulus) to the activity patterns. The eigenvectors (not shown) are nearly identical for the first component ( $x$-axis). Therefore the 
A

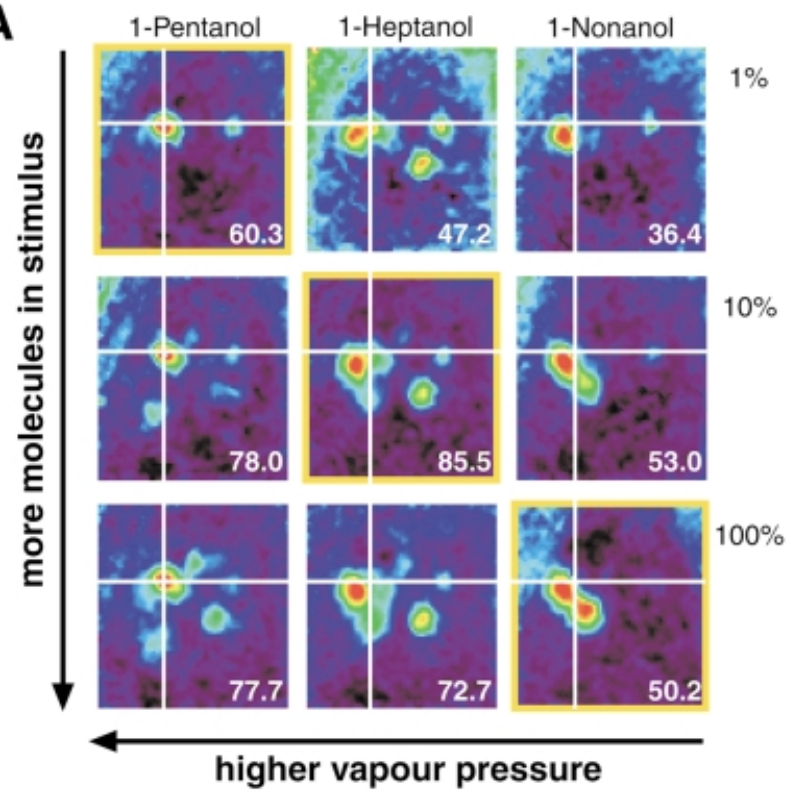

B

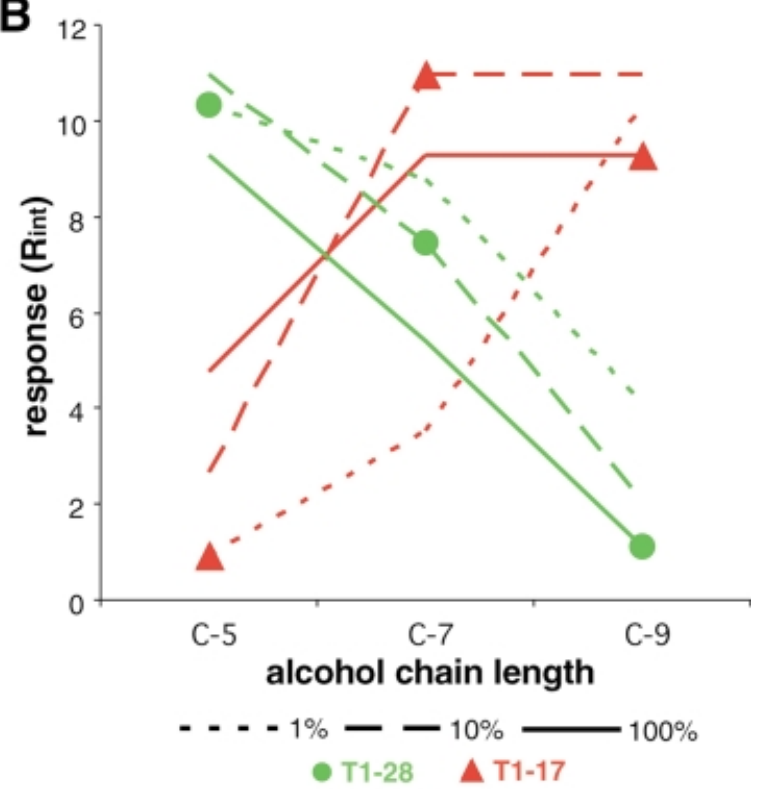

FIG. 4. A change in vapour pressure does not interfere with the specificity of activity patterns. (A) False-colour-coded calcium signals during stimulation with different primary alcohols at three concentration levels. For colour-code see Fig. 7. Cumulative AL activity is given at the bottom right of each frame. The crosswires mark the glomerulus T1-28. The pattern is widely preserved over the concentration levels. (B) Responses of two identified glomeruli to three different primary alcohols at the same concentration levels tested as those in A. A stimulus with 1\% 1-pentanol corresponds to a stimulus with $10 \%$ 1-heptanol and $100 \% 1$ nonanol in terms of numbers of molecules per volume of air. These concentrations are marked with symbols. It is apparent that T1-28 responds strongest to 1pentanol, and T1-17 to longer chain lengths irrespective of the stimulus concentrations tested.

arrangement of the alcohols along the $x$-axis corresponds to their cumulative AL activity. This arrangement reflects the decrease of effective vapour pressure (Fig. 3B), and is therefore not related to a structure-correlated receptor-odour interaction. The other two components reflect specific glomerular contrasts. The second component ( $y$-axis) opposes the responses of glomeruli with strong chain length dependence for shorter (C-5, C-6: T1-28 and T1-38) to those for longer (C-9, C-10: T1-17 and T1-33) chain length ranges. The arrangement along the $z$-axis is due to glomeruli which are optimally stimulated by intermediate chain length. Therefore, rising carbon chain lengths are arranged as a semicircle (Fig. 5B). This arrangement indicates that activity pattern similarity is correlated with carbon chain length difference within a functional group.

\section{Are there any glomeruli which specifically respond to functional groups of odour molecules?}

Figure 6A presents the responses of four identified glomeruli during stimulation with different hydrocarbons. We did not find any glomerulus that specifically responds to functional groups independently of carbon chain length. Each evaluated glomerulus shows similar chain length dependence independently of chemical families (Fig. 6A) with the exception of the alkanes, which are ineffective as stimulus for most glomeruli. The maximal glomerular response is not restricted to a peak, but rather to a range of chain lengths (compare with Fig. 5A). We found that the optimal chain length can vary between one and three carbon atoms for the different chemical classes. Furthermore, the different functional groups elicit differences in the maximum response intensities. For example in Fig. 6A, glomerulus T1-17 reveals a maximal response when stimulated with alcohols in a range of seven to eight carbon atoms, whereas ketones and aldehydes elicit lower activity with a peak response at eight or nine carbon atoms. Glomerulus T1-42 is strongly activated for ketones with a short carbon chain and glomerulus T1-52 for aldehydes with an intermediate chain length. Alkanes elicit a strong response only in glomerulus T3-45, which also shows chain length-dependent properties.

A PCA of the glomerular representations for the different hydrocarbons shows good separation for alkanes but only incomplete clustering to their chemical classes for the different alcohols, aldehydes or ketones (Fig. 6B). This confirms that there are no glomeruli for functional groups. Most of the variance $(88.3 \%$ ) can be represented in a three-dimensional space. All eigenvectors show similar values for the first component (eigenvectors not shown). This axis separates the chemical families to their evoked cumulative activity into two groups (alcohols and alkanes on the one side, and aldehydes and ketones on the other). However, differences in activity patterns of functional groups do not depend on stimulus concentration. The correlations do not increase between the activity patterns when alcohols, aldehydes and ketones with nearly identical effective concentrations are applied (data not shown). The contrast of glomerulus T3-45 with the other glomeruli in the second component ( $y$-axis) leads to clustering of the alkanes, because they are the only stimuli which elicit a strong response in T3-45 but no response in all other glomeruli. The $z$-axis (third component) opposes the response of glomeruli that are maximally stimulated with shorter (T1-28, 36) against intermediate (T1-29, 35, 38, 52) chain lengths. Therefore, alcohols, aldehydes and ketones are arranged according to their chain lengths. Hydrocarbons with chain lengths of eight to 10 carbon atoms form a cluster in the $y-z$-plot (with the exception of the alkanes). This means that for long chain lengths the correlation between alcohols, aldehydes and ketones is higher, which is confirmed by direct comparison of these values (Fig. 6C). The correlation decreases for shorter molecules. A stronger and always constant correlation is seen when comparing molecule length neighbours having the same functional group (solid line in Fig. 6C). 
A

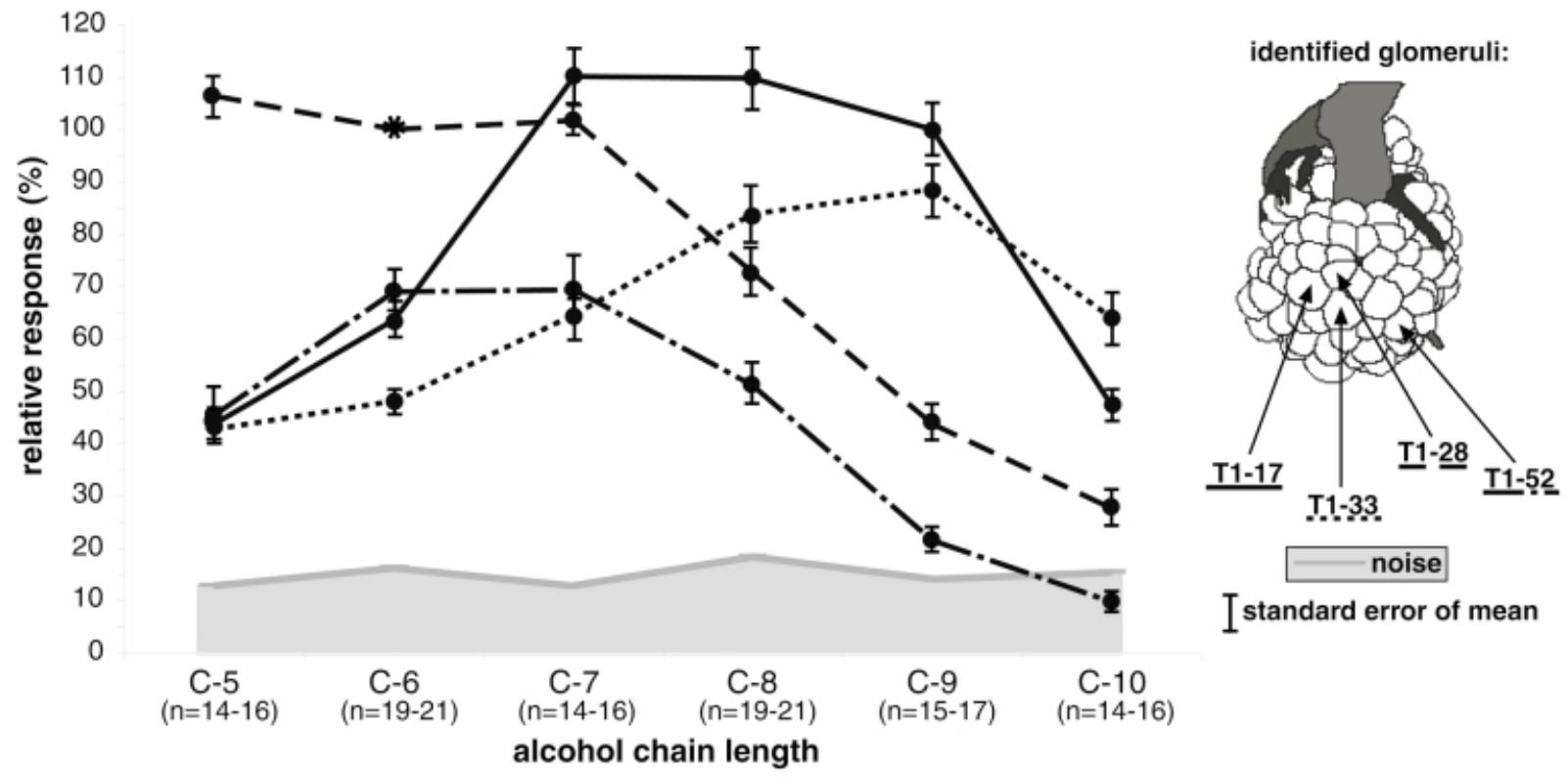

B

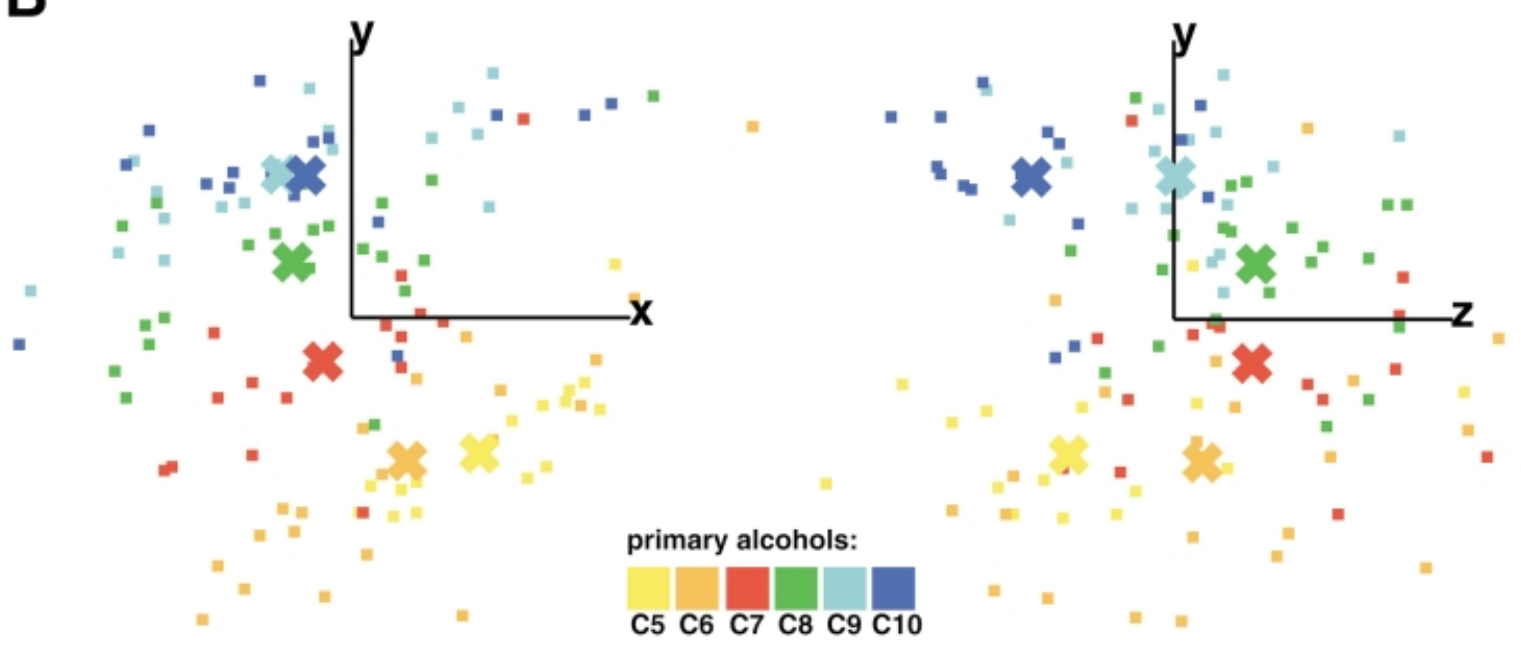

FIG. 5. Responses to primary alcohols with a range of five to 10 carbon atoms. (A) Averaged responses ( \pm SEM) from four identified glomeruli to different primary alcohols. Single measurements are normalized to the response intensity of glomerulus T1-28 to 1-hexanol (labelled with asterisk, no error bar). The noise limit is indicated in grey. The number of averaged measurements is shown below the odours. Glomerular properties can be classified into the following three groups: (i) strong activation for short carbon chain lengths (glomerulus T1-28); (ii) maximum activity when stimulated with an intermediate chain (glomeruli T1-17 and T152); and (iii) increase of activity with longer carbon chain (glomerulus T1-33). All responses with one exception are above the noise limit (see experimental procedures). (B) Principal component analysis of the glomerular responses, performed on all measured individuals for all tested primary alcohols. The single measurements are shown as dots; their mean values are marked with ' $X$ 's. Each colour indicates a specific carbon chain length. Note that the alcohols are arranged following their chain length. Most overlaps of single measurements are restricted to neighbouring chain length ranges.

FIG. 6. Glomerular responses to hydrocarbons differing in their functional groups and chain lengths. (A) Averaged responses of four identified glomeruli to primary alcohols, aldehydes, secondary ketones and alkanes from five to 10 carbon atoms. Missing symbols indicate responses within noise limits. When comparing glomerular responses to hydrocarbons with identical chain length but differing in their functional groups, large differences are obvious at first sight, but consideration of the complete chain length range (C5-10) reduces these differences. All glomeruli show a specific chain length dependence that is independent of the functional group. However, the intensity and maximal chain length range of the responses can differ between the different chemical classes. (B) Principal component analysis of the averaged activity patterns for different hydrocarbons. The colours indicate the chemical class, the numbers the chain length of the odours. A clustering of the alkanes is resolved along the $y$-axis. The other chemical classes could be arranged according to increasing chain length along the $y$-and $z$-axes. There is no separation of the functional groups. (C) Correlation of response patterns to primary alcohols, aldehydes and secondary ketones of identical chain length (dashed, dotted and dash-dotted line), and averaged correlation of neighbouring chain lengths of identical functional groups (solid line). Correlation between functional groups increases with carbon chain lengths. Molecules with neighbouring chain length always elicit highly correlated activity patterns. Note that 2-heptanone is peculiar in that it differs from both heptanal and 1-heptanol. 


\section{A special case for 2-heptanone}

2-Heptanone has a particularly specific activity pattern which differs from heptanal and heptanol more than the functional groups differ at other chain lengths. This may be due to the particular role of this odour as a repellent scent-mark which is used by honeybees to mark visited flowers, and thus prevent a precocious re-visit (Giurfa \& Núñez, 1992; Giurfa, 1993). The uniqueness of 2-heptanone is only apparent by comparing the patterns of the glomeruli using correlation analysis (Fig. 6C) and not by inspection of Fig. 2. This emphasizes

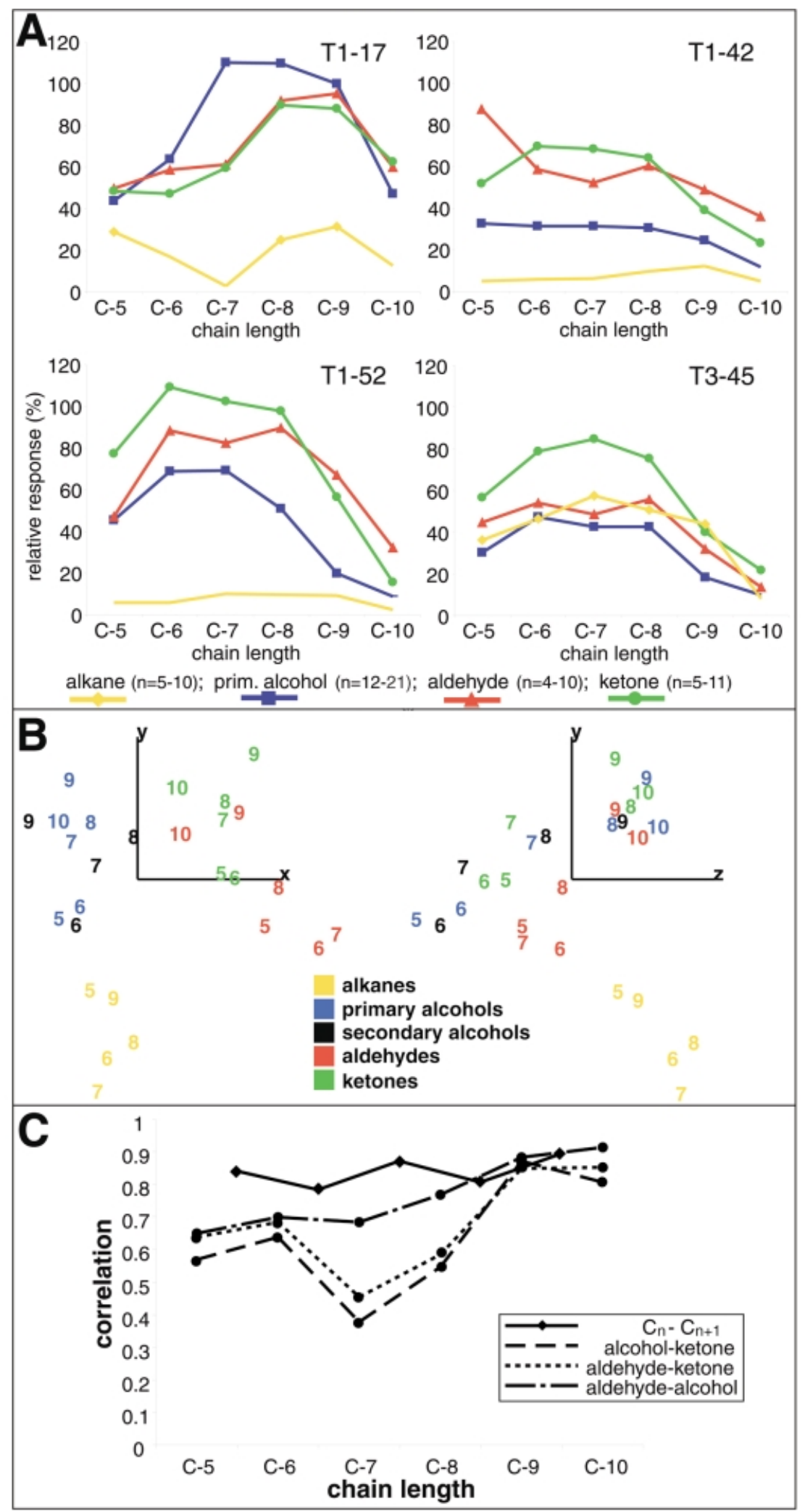


the importance of looking at the entire pattern of activated glomeruli for the olfactory code.

\section{Position of the functional group: primary and secondary alcohols}

The position of the functional group at the odour molecule influences the glomerular encoding of the chain length. Secondary and primary alcohols activate the same sets of glomeruli, e.g. glomerulus T1-28 for short, T1-52 for intermediate and T1-33 for long molecules. However, the response patterns to secondary alcohols are generally intermediate between the corresponding primary alcohol and the primary alcohol lacking one carbon atom in the chain (see the response intensities of glomerulus T1-28 in Fig. 2; best responses are to primary alcohols C5-C6-C7, and to secondary alcohols C6-C7-C8).

This is evident in the PCA in Fig. 6B, where the secondary alcohols are interspersed within the sequence of primary alcohols. Also the correlation matrix supports this suggestion (Table 1). Here, each secondary alcohol shows its strongest correlation with the corresponding and the shortened primary alcohol rather than with its secondary alcohol neighbours. For example, the response to 2heptanol is correlated to 1-heptanol and 1-hexanol with 0.93 and 0.90, respectively, but to 2-hexanol and 2-octanol with 0.86 and 0.88 , respectively (printed as a reading example in bold letters in Table 1).

\section{Spatial shift in the activity patterns for hydrocarbons}

Is there any relationship between the glomerular response range and its position in the AL? Representations of the spatial glomerular code (Fig. 2) can be displayed using a schematic view of the AL (Fig. 7A). The colour code in Fig. 7 gives the relative response intensity corresponding to the circle sizes in Fig. 2. Alkanes evoked activity almost exclusively in glomerulus T3-45. Its chain length-dependent properties are not visible in this figure because data were normalized (compare with the absolute response intensities of glomerulus T3-45 in Fig. 6A). The activity patterns elicited by primary alcohols (Fig. 7B) change from a central component for short carbon chains dominated by T1-28 to a lateral-diagonal pattern for longer chain lengths (glomeruli T1-17 and T1-33). Patterns evoked by aldehydes or ketones (Fig. 7B) show similar sliding alterations. Increasing chain lengths lead to activity patterns with a continuous shift within functional groups, indicating that odours with similar chain lengths elicit similar spatial activity patterns.

Interestingly, the maximally excited glomeruli are generally localized near each other. Furthermore, the continuous pattern shift is always a movement toward neighbouring glomeruli. The increasing similarities between functional groups in the spatial pattern for increasing chain length are again apparent in this analysis (compare with Fig. 6B and C).

\section{Discussion}

Morphological mapping of the AL after calcium imaging allowed us to evaluate activity patterns at the level of identified glomeruli. Therefore, we can investigate how molecular features of an odour molecule influence glomerular responses. In order to approach the rules of chemical structure representation we tested a wide range of hydrocarbons differing systematically in carbon chain length and functional group. Here we describe the molecular receptive range of 38 morphologically identified glomeruli with an odour space spanning aldehydes, ketones, alcohols, carboxylic acids and alkanes varying in chain length from $\mathrm{C} 5$ to $\mathrm{C} 10$.

\section{Methodological considerations}

In this work, the intracellular calcium-sensitive dye was bathapplied, which leads to all cells in the AL picking up the dye. Therefore, we do not know the relative proportion of the different AL cells (ORNs, LINs and PNs) contributing to the signal. The major contribution appears to be from ORNs (Galizia et al., 1998). In two recent papers where single-cell recordings could be related to identified glomeruli in the macroglomerular complex of the moth Heliotis virescens, the response properties of afferent ORNs (Berg et al., 1998) match those of the PNs (Vickers et al., 1998). Therefore, in each glomerulus the molecular receptive response profile of PNs may be closely related to the molecular receptive ranges of the ORNs innervating the glomerulus, and the contribution of both to the calcium activity measured in this paper may be similar. This would imply an isomorphism between the across-glomeruli code for each odour, and the across-fibre code in the PNs. Activity in LINs, however, may be more widespread in

TABLE 1. Correlation of response patterns of primary and secondary alcohols

\begin{tabular}{|c|c|c|c|c|c|c|c|c|c|c|}
\hline Variable & \multicolumn{6}{|c|}{ Primary alcohols } & \multicolumn{4}{|c|}{ Secondary alcohols } \\
\hline C-5 & 1.00 & & & & & & & & & \\
\hline C-6 & 0.84 & 1.00 & & & & & & & & \\
\hline $\mathrm{C}-7$ & 0.70 & 0.80 & 1.00 & & & & & & & \\
\hline C-8 & 0.45 & 0.53 & 0.91 & 1.00 & & & & & & \\
\hline \multicolumn{11}{|c|}{ Secondary alcohols } \\
\hline C-6 & 0.89 & 0.91 & 0.73 & 0.41 & 0.23 & 0.19 & 1.00 & & & \\
\hline $\mathrm{C}-7$ & 0.81 & 0.90 & 0.93 & 0.75 & 0.58 & 0.48 & 0.86 & 1.00 & & \\
\hline $\mathrm{C}-8$ & 0.60 & 0.75 & 0.94 & 0.87 & 0.75 & 0.68 & 0.67 & 0.88 & 1.00 & \\
\hline C-9 & 0.47 & 0.46 & 0.88 & 0.95 & 0.91 & 0.74 & 0.41 & 0.73 & 0.82 & 1.00 \\
\hline
\end{tabular}

Each row and column represents one primary or secondary alcohol arranged according to increasing chain length. The correlations between the activity patterns decrease with increasing chain length distance of the odours. Note that the secondary alcohols show less correlation to their secondary alcohol neighbours than to the corresponding or shortened primary alcohol. Correlation of 2-heptanol to structurally closely related molecules is shown in bold (for details see text). 


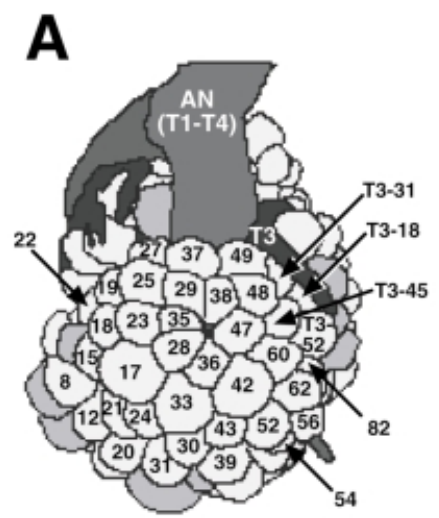

B
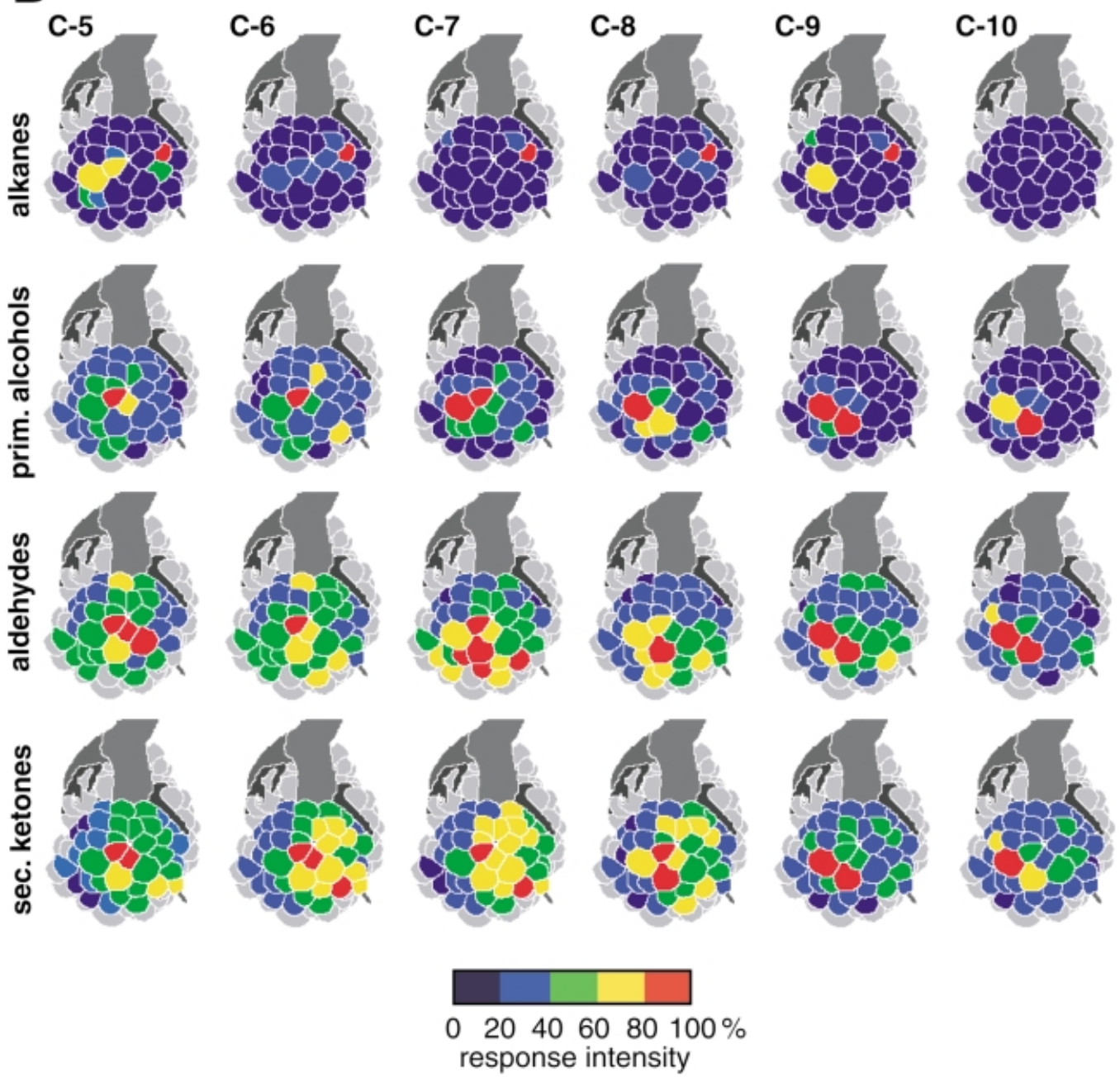

FIG. 7. The spatial activity patterns of several hydrocarbons depicted with a graphical AL. (A) Schematic AL derived from the standard atlas. The 38 identified glomeruli, which could be recognized in the measurements, are labelled. The antennal nerve enters from the top. All glomeruli without tract labelling are innervated by T1. Glomeruli identified in only a few animals are shown in dark grey. (B) Activity patterns during stimulation with alkanes, primary alcohols, aldehydes and secondary ketones with a chain length of five to 10 carbon atoms. Alkanes elicit a strong response only in glomerulus T3-45. The spatial responses to alcohols turn from a strong activation of glomerulus T1-28 to a lateral diagonal pattern (glomeruli T1-17 and T1-33) with increasing chain length. Aldehydes and ketones effect similar pattern shifts with increasing chain lengths. Both elicit stronger overall responses compared with alcohols and alkanes.

the AL, leading to an underlying global activity in many glomeruli, as is apparent in the responses to ketones (Fig. 2). However, no conclusive attribution to the cellular origin of the signal can be made with the methods applied. Therefore, the data are best interpreted as a measure of the integrated, overall activity in each glomerulus.
The data shown in this paper are based on slow calcium measurements ( 2 frames/s) of the entire neuropil. Dynamic aspects, e.g. the sequence of glomerular activation (Joerges et al., 1997; Galizia et al., 1997) or fast temporal components (e.g. Laurent, 1996), which are likely to be part of the complete olfactory code, were ignored in this study. 
The results show highly conserved glomerular odour representation among individuals in the AL of honeybees (colour code in Fig. 2). Therefore, we can average the glomerular odour representation of several individuals and approach the spatial olfactory code. Nevertheless, within the stereotypical patterns there is a variable component; the calcium responses in the $\mathrm{AL}$ are influenced by appetitive training in the honeybee (Faber et al., 1999). Furthermore, developmental plasticity has been shown to affect the size of honeybee glomeruli (Winnington et al., 1996; Sigg et al., 1997). We measured large sampling sizes in order to reduce the effects of learning and plasticity which may have occurred before the measurements.

\section{Odours with no response}

The spatial olfactory code is determined by the total of all glomeruli and spans a virtually unlimited number of odour representations. Figure 2 presents a subset of this code. In this 'across-glomeruli code' each odour is coded in the activity of several glomeruli, and each glomerulus participates in the code of several odours (Joerges et al., 1997 and Fig. 2). We could not measure any response to carboxylic acids and long chain alkanes (Fig. 2). For carboxylic acids this could be due to their low vapour pressures (Fig. 3B). However, the range of vapour pressure of the hydrocarbons overlaps with that of alcohols, which elicit strong responses (Fig. 3C). For the alkanes, the absence of electronegativity (see Results), or their hydrophobic properties could reduce receptor interactions and thus glomerular responses. The queen's mandibular glands secrete a wide range of alkanes and carboxylic acids (Engels et al., 1997) which provide particularly important signals for queen-drone (Gary, 1962) as well as for queenworker (Kaminsky et al., 1990) interactions. Additionally, both electrophysiological recordings of a mushroom body extrinsic neuron, the Pe1 neuron, and behavioural experiments show that honeybees can perceive and discriminate the tested carboxylic acids (Vareschi, 1971; Rybak \& Menzel, 1998). Therefore, the lack of glomeruli responding does not indicate poor perception of these odours. They are probably coded in glomeruli not included in our sample.

\section{No single glomerulus contains the 'code' for a functional group}

It could have been possible that a glomerulus responded to a functional group over a very wide range of carbon chain lengths, and thus reported the presence of, e.g. aldehyde. We did not find any glomerulus that was specifically activated by any of the chemical groups tested. Information about the functional groups is therefore only present in the entire glomerular pattern. As shown in Fig. 6A, each functional group elicits a peak response to a particular chain length irrespective of its chemical family, but with specific maximal intensity. For example, glomerulus T1-17 is preferentially activated by alcohols, glomeruli T1-42 or T1-52 and T3-45 by aldehydes or ketones, respectively. Therefore, the information present in the pattern is still sufficient to unambiguously identify all odours tested, so that there is, with the information given by the measured glomeruli, no need for a functional group-specific glomerulus.

This lack of glomeruli specific for functional groups is in contrast to what is found in mammals (Johnson et al., 1998). This may reflect basic differences between the AL and the olfactory bulb. The latter, with $\sim 1000$ glomeruli, may have much more narrowly tuned glomerular response profiles than the honeybee with 160 glomeruli. The broader tuning in honeybees may be due to differences in the response properties of olfactory receptors, or because olfactory glomeruli may be innervated by more than one ORN-type in insects (Vosshall et al., 1999).

\section{Molecular receptive range depends on carbon chain length}

The carbon chain length is the main common variable influencing the glomerular response profile to hydrocarbons with different functional groups. More than $50 \%$ of the identified glomeruli reflect chain length in their responses. However, no single glomerulus contains the 'code' for chain length as this information always depends on comparing glomeruli with different response peaks. The multidimensional ranges occupied by the measurements of single animals show some overlap, indicating that chemically close odours evoke similar activity patterns (Fig. 5B). Behavioural experiments about honeybee odour discrimination suggest that these odours may indeed be perceptually close; confusions only occur between odours with neighbouring chain lengths (Laska et al., 1999).

\section{Implications for odorant-receptor interaction}

The excitation properties of ORNs are the result of interactions between their receptor proteins and an odour molecule. In vertebrates, ORNs expressing the same receptor protein converge onto single glomeruli and each ORN expresses only one olfactory receptor gene (Ressler et al., 1994; Vassar et al., 1994; Mombaerts et al., 1996; Malnic et al., 1999). Therefore, the glomeruli would be isomorphic to ORN-families, and the molecular receptive range of the glomeruli would allow conclusions about the receptive range of ORNs. This isomorphism has not been shown in insects, where far fewer glomeruli (160 in honeybees against $\sim 1000$ in each half bulb of the mouse) could mean that more than one receptor type innervates each glomerulus, as has been suggested for Drosophila (Clyne et al., 1999; Vosshall et al. 1999). Still, the glomerular response would be a consequence of its innervation pattern, as modified by the ALnetwork, and allow conclusions to be drawn about the odour-receptor interactions occurring at the periphery. In the present study we show that carbon chain length of odour molecules represents an important criterion for receptor interactions. This interaction is unsharp as a certain chain length elicits the strongest, but not the only, response (Fig. 5A). Broad stimulus-response profiles have been reported for receptor cells (Altner et al., 1977; Sicard \& Holley, 1984; Sato et al., 1994; Malnic et al., 1999; Duchamp-Viret et al., 1999) though other data argue for more restricted response spectra (Zhao et al., 1998; Krautwurst et al., 1998).

The mechanisms by which receptors become selective are still unclear. We show here that these mechanisms must be a function of the entire odorant molecule, and not just of an epitope. This is also shown by the increasing similarities of the patterns for alcohols, aldehydes and ketones with increasing chain length (Figs 6B and C, and 7B). The hydrophobic alkyl-residue overshadows the influence of the functional group with increasing chain length. Because olfactory receptors have been recently cloned in Drosophila (Clyne et al., 1999; Vosshall et al., 1999), it should soon be possible to measure the odorant response profiles of identified olfactory receptor proteins. The 'lock and key' principle is supported by the strong correlation between odour quality and molecular shape (Amoore, 1964, 1971), and specific anosmia for odours with similar structures (Amoore, 1967), and suggests the notion of 'odotopes' acting as epitopes in the antibody-antigen interactions. Alternatively, the olfactory system could work as a spectroscope, where the vibrations of the molecules (Wright, 1977) would be measured by a vibrational transduction mechanism (Turin, 1996). Hydrocarbons with even chain lengths have vibration spectra differing from those with odd chain lengths (Turin, 1996); in our measurements, we never found 'odd' - or 'even'specific response properties, which would argue against the importance of these vibrational frequencies. 


\section{Implications for the $A L$ network}

The sliding shift of the glomerular response properties evoked at different carbon chain lengths of the odour molecules results in a continuity of odour representation in the AL. To ensure a specific recognition they have to be sharpened at 'higher' processing stations. A possible realization of such a specific 'read-out' would be the narrowing of glomerular response profiles through a lateral inhibition of the PNs. Indeed, single-cell measurements of honeybee PNs reveal both excitatory and inhibitory responses to odours (Abel, 1997). Results from single-cell measurements in rabbits show that mitral cells are distinctly excited by aldehydes of a limited range of chain length and inhibited by aldehydes with neighbouring chain length (Yokoi et al., 1995). This mechanism evidently sharpens the molecular receptive range of individual mitral cells and is also plausible for the PNs in the honeybee. This computation would be performed by LINs connecting the glomeruli. The close neighbouring relationship of chain length-encoding glomeruli, e.g. T1-17, T1-28, T1-33 and T1-36 (Fig. 7) would suggest that this chemical lateral inhibition is achieved by spatial lateral inhibition. In this way the AL could minimize the total wiring length of its interneurons.

The AL of honeybees is compartmentalized into four subunits, defined by the four tracts of the antennal nerve T1-4. We have measured $34(\sim 50 \%)$ of the glomeruli in $\mathrm{T} 1$, and four $(\sim 6 \%)$ in $\mathrm{T} 3$. Thus, $\mathrm{T} 1$ is well covered while $\mathrm{T} 3$ is only poorly represented, and the other subunits are not represented at all (Fig. 2). This prohibits conclusions about the role of these subcompartments. That there may be a separation in terms of odours encoded is suggested by the fact that we only found responses to alkanes in T3. However, other odours elicit responses both in T1 and T3 (e.g. aldehydes, secondary ketones, and other odours not shown in this paper). This indicates that $\mathrm{T} 1$ and $\mathrm{T} 3$ are not mutually exclusive in their response characteristics.

Future experiments will investigate the complete glomerular olfactory code of the honeybee by broadening the analysed odour repertoire, and measuring activity patterns of the entire AL. Furthermore, we will combine intracellular recordings from LINs and PNs with calcium imaging, and test interneurons with the same odorants as were used in the imaging experiments.

\section{Acknowledgements}

We are grateful to Randolf Menzel for unlimited support. Thanks also to Frank Müller (Institute for 'Angewandte Zoologie', FU Berlin) for the GC-analysis of the odorants, to Anke Friedrich, Matthias Laska, Till Faber, Dirk Müller and Raúl Rojas for comments on the manuscript, and to Mary Wurm and Nina Kühn for correcting the English.

\section{Abbreviations}

AL, antennal lobe; DMSO, dimethylsulphoxide; LIN, local interneuron; ORN, olfactory receptor neuron; PCA, principal component analysis; PN, projection interneuron; $R_{\mathrm{int}}$, response of single glomeruli as integral of the time interval covering frames 9-19; $R_{\mathrm{int}}(\max )$, maximal $R_{\text {int }}$ of all glomeruli as response of one odour; T1-4, olfactory tracts $1-4$.

\section{References}

Abel, R. (1997) Das olfaktorische System der Honigbiene: Elektrophysiologische und morphologische Charakterisierung von Antennallobus Neuronen und deren Beteiligung beim olfaktorischen Lernen. PhD thesis, Freie Universität, Berlin.

Altner, H., Sass, H. \& Altner, I. (1977) Relationship of structure and function of antennal chemo-, hygro-, and thermoreceptive sensilla in Periplaneta americana. Cell Tissue Res., 176, 389-405.

Amoore, J.E. (1964) The stereochemical theory of odor. Sci. Am., 210, 42-49. Amoore, J.E. (1967) Specific anosmia: a clue to the olfactory code. Nature, 214, 1095-1098.
Amoore, J.E. (1971) Stereochemical and vibrational theories of odor. Nature, 233, 270-271.

Berg, B.G., Almaas, T.J., Bjaalie, J.G. \& Mustaparta, H. (1998) The macroglomerular complex of the antennal lobe in the tobacco budworm moth Heliothis virescens: specified subdivision in four compartments according to information about biologically significant compounds. $J$. Comp. Neurophysiol. A, 183, 669-682.

Boeckh, J., Distler, P., Ernst, K.D., Hösl, M. \& Malun, D. (1990) Olfactory bulb and antennal lobe. In Schild, D. (ed.), Chemosensory Information Processing. Springer, Berlin, pp. 201-227.

Cinelli, A.R., Hamilton, K.A. \& Kauer, J.S. (1995) Salamander olfactory bulb neuronal activity observed by video rate, voltage-sensitive dye imaging. III. Spatial and temporal properties of responses evoked by odorant stimulation. J. Neurophysiol., 73, 2053-2071.

Clyne, P.J., Warr, C.G., Freeman, M.R., Lessing, D., Kim, J. \& Carlson, J.R. (1999) A novel family of divergent seven-transmembrane proteins: candidate odorant receptors in Drosophila. Neuron, 22, 327-338.

Dobson, H.E.M. (1994) Floral volatiles in insect biology. In Bernays, F.A., (ed.), Insect-Plant Interaction, Vol. 5. CRC Press, Boca Raton, pp. 48-81.

Duchamp-Viret, P., Chaput, M.A. \& Duchamp, A. (1999) Odor response properties of rat olfactory receptor neurons. Science, 284, 2171-2174.

Engels, W., Rosenkranz, P., Adler, A., Taghizadeh, T.L., Lübke, G. \& Francke, W. (1997) Mandibular gland volatiles and their ontogenetic patterns in queen honey bees, Apis mellifera carnica. J. Insect Physiol., 43, 307-313.

Esslen, J. \& Kaissling, K.-E. (1976) Zahl und Verteilung antennaler Sensillen bei der Honigbiene (Apis mellifera L.). Zoomorphol., 83, 227-251.

Faber, T., Joerges, J. \& Menzel, R. (1999) Associative learning modifies neural representations of odors in the insect brain. Nature Neurosci., 2, 74 78.

Flanagan, D. \& Mercer, A.R. (1989) An atlas and 3-D reconstruction of the antennal lobes in the worker honey bee, Apis mellifera L. (Hymenoptera: apidae). Int. J. Insect Morphol. Embryol., 18, 145-159.

Friedrich, R.W. \& Korsching, S.I. (1997) Combinatorial and chemotopic odorant coding in the zebrafish olfactory bulb visualised by optical imaging. Neuron, 18, 737-752.

Friedrich, R.W. \& Korsching, S.I. (1998) Chemotopic, and noncombinatorial odorant representation in the olfactory bulb revealed using a voltagesensitive dye. J. Neurosci., 18, 9977-9988.

Frisch, K.V. (1967) The Dance Language and Orientation of Bees. Harvard University Press, Cambridge.

Galizia, C.G., Joerges, J., Kuettner, A., Faber, T. \& Menzel, R. (1997) A semiin-vivo preparation for optical recording of the insect brain. J. Neurosci. Meth., 76, 61-69.

Galizia, C.G., Nägler, K., Hölldobler, B. \& Menzel, R. (1998) Odor coding is bilaterally symmetrical in the antennal lobes of honeybees (Apis mellifera). Eur. J. Neurosci., 10, 2964-2974.

Galizia, C.G., McIlwrath, S.L. \& Menzel, R. (1999a) A digital 3D atlas of the honeybee antennal lobe based on optical sections acquired using confocal microscopy. Cell Tissue Res., 295, 383-394.

Galizia, C.G., Sachse, S., Rappert, A. \& Menzel, R. (1999b) The glomerular code for odor representation is species-specific in the honeybee Apis mellifera. Nature Neurosci., 2, 473-478.

Gary, N.E. (1962) Chemical mating attractants in the honeybee. Science, 136, 773-774.

Gascuel, J. \& Masson, C. (1991) A quantitative ultrastructural study of the honeybee antennal lobe. Tissue Cell, 23, 341-355.

Giurfa, M. \& Núñez, J.A. (1992) Honeybees mark with scent and reject recently visited flowers. Oecologia, 89, 113-117.

Giurfa, M. (1993) The repellent scent-mark of the honeybee Apis mellifera ligustica and its role as communication cue during foraging. Insectes Sociaux, 40, 59-67.

Hass, H.B. \& Newton, R.F. (1975) Correction of boiling points to standard pressure. In Weast, R.C. (ed.), Handbook of Chemistry and Physics. CRC Press, Cleveland, OH, USA, pp. D176-D177.

Hauptmann, S. (1987) Polare Substituenteneffekte. In Hauptmann, S. (ed.), Organische Chemie. VEB Deutscher für Grundstoffindustrie, Leipzig, pp. $179-183$.

Imamura, K., Mataga, N. \& Mori, K. (1992) Coding of odor molecules by mitral/tufted cells in rabbit olfactory bulb. I. Aliphatic compounds. $J$. Neurophysiol., 68, 1986-2002.

Joerges, J., Kuettner, A., Galizia, C.G. \& Menzel, R. (1997) Representations of odors and odor mixtures visualised in the honeybee brain. Nature, 387, $285-288$.

Johnson, B.A., Woo, C.C. \& Leon, M. (1998) Spatial coding of odorant 
features in the glomerular layer of the rat olfactory bulb. J. Comp. Neurol., 393, 457-471.

Kaissling, K.-E. (1995) Single unit and electroantennogram recordings in insect olfactory organs. In Spielman, A. I. \& Brand J. G. (eds), Experimental Cell Biology of Taste and Olfaction: Current Techniques and Protocols. CRC Press, Boca Raton, pp. 361-377.

Kaminsky, L.A., Slessor, K.N., Winston, M.L., Hay, N.W. \& Borden, J.H. (1990) Honeybee response to queen mandibular pheromone in laboratory bioassays. J. Chem. Ecol., 16, 841-850.

Krautwurst, D., Yau, K.-W. \& Reed, R.R. (1998) Identification of ligands for olfactory receptors by functional expression of a receptor library. Cell, $\mathbf{9 5}$, 917-926.

Laska, M., Galizia, C.G., Giurfa, M. \& Menzel, R. (1999) Olfactory discrimination ability and odor structure-activity relationships in honeybees. Chem. Senses, 24, 429-438.

Laurent, G. (1996) Dynamical representation of odors by oscillating and evolving neural assemblies. Trends Neurosci., 19, 489-496.

Malnic, B., Hirono, J., Sato, T. \& Buck, L.B. (1999) Combinatorial receptor codes for odors. Cell, 96, 713-723.

Mombaerts, P., Wang, F., Dulac, C., Chao, S.K., Nemes, A., Mendelson, M. Edmonson, J. \& Axel, R. (1996) Visualizing an olfactory sensory map. Cell, 87, 675-686.

Mori, K., Mataga, N. \& Imamura, K. (1992) Differential specificities of single mitral cells in rabbit olfactory bulb for a homologous series of fatty acid odor molecules. J. Neurophysiol., 67, 786-789.

Ressler, K.J., Sullivan, K.J. \& Buck, L.B. (1994) Information coding in the olfactory system: evidence for a stereotyped and highly organized epitope map in the olfactory bulb. Cell, 79, 1245-1255.

Rybak, J. \& Menzel, R. (1998) Integrative properties of the Pe1 neuron, a unique mushroom body output neuron. Learning Memory, 5, 133-145.

Sachse, S., Rappert, A., Galizia, C.G. \& Menzel, R. (1998) Alcohols of similar carbon chain length elicit similar activity patterns in the honeybee brain. Eur. J. Neurosci., 10 (Suppl. 10), 358.

Sato, T., Hirono, J., Tonoike, M. \& Takebayashi, M. (1994) Tuning specificities to aliphatic odorants in mouse olfactory receptor neurons and their local distribution. J. Neurophysiol., 72, 2980-2989.
Shepherd, G.M. (1991) A model system for computational neuroscience. In Davies, J. \& Eichenbaum, H. (eds), Olfaction. MIT Press, Cambridge, MA, USA, pp. 3-41.

Sicard, G. \& Holley, A. (1984) Receptor cell responses to odorants: similarities and differences among odorants. Brain Res., 292, 283-296.

Sigg, D., Thompson, C.M. \& Mercer, A.R. (1997) Activity-dependent changes to the brain and behavior of the honeybee, Apis mellifera (L.). J. Neurosci., 17, 7148-7156.

Suzuki, H. (1975) Antennal movements induced by odor and central projection of the antennal neurons in the honeybee. J. Insect Physiol., 21, 831-847.

Turin, L. (1996) A spectroscopic mechanism for primary olfactory reception. Chem. Senses, 21, 773-791.

Vareschi, E. (1971) Duftunterscheidung bei der Honigbiene-EinzelzellAbleitungen und Verhaltensreaktionen. Z. Vergl. Physiol., 75, 143-173.

Vassar, R., Chao, S.K., Sitcheran, R., Nunez, J.M., Vosshall, L.B. \& Axel, R. (1994) Topographic organization of sensory projections to the olfactory bulb. Cell, 79, 981-991.

Vosshall, L.B., Amrein, H., Morozov, P.S., Rzhetsky, A. \& Axel, R. (1999) A spatial map of olfactory receptor expression in the Drosophila antenna. Cell, 96, 725-736.

Vickers, N.J., Christensen, T.A. \& Hildebrand, J.G. (1998) Combinatorial odor discrimination in the brain: attractive and antagonist odor blends are represented in distinct combinations of uniquely identifiable glomeruli. $J$. Comp. Neurol., 400, 35-56.

Winnington, A.P., Napper, R.M. \& Mercer, A.R. (1996) Structural plasticity of identified glomeruli in the antennal lobes of the adult worker honeybee. $J$. Comp. Neurol., 365, 479-490.

Wright, R.H. (1977) Odor and molecular vibration: neural coding of olfactory information. J. Theor. Biol., 64, 473-502.

Yokoi, M., Mori, K. \& Nakanishi, S. (1995) Refinement of odor molecule tuning by dendrodendritic synaptic inhibition in the olfactory bulb. Proc. Natl Acad. Sci. USA, 92, 3371-3375.

Zhao, H., Ivic, L., Otaki, J.M., Hashimoto, M., Mikoshiba, K. \& Firestein, S. (1998) Functional expression of a mammalian odorant receptor. Science, 279, 237-242. 